\title{
Incidence des caractéristiques individuelles et des caractéristiques du contexte sur les nouveau-nés de faible poids : une étude d'observation au Québec
}

\author{
N. Savard, M. Sc. (1, 2); P. Levallois, M.D. (1, 2, 3); L. P. Rivest, Ph. D. (4); S. Gingras, M. Sc. (2)
}

Cet article a fait l'objet d'une évaluation par les pairs.

\section{Résumé}

Introduction : Nous avons analysé les liens entre certaines variables contextuelles, certaines caractéristiques des mères et le risque de donner naissance à des nouveau-nés très petits pour l'âge gestationnel (TPAG) au Québec entre 2000 et 2008.

Méthodologie : Les variables liées au contexte ont été tirées de l'Enquête sur la santé dans les collectivités canadiennes, du Recensement canadien et du Registre des naissances du Québec. Les variables individuelles provenaient aussi du Registre des naissances du Québec. Les rapports de cotes (RC), ajustés selon l'âge de la mère, la scolarité, la parité, l'état matrimonial et le pays de naissance, ont été évalués au moyen de modèles de régression logistique multiniveau (par des équations d'estimation généralisées).

Résultats : Les naissances survenues dans les quartiers présentant une proportion élevée de résidents sédentaires ( $\mathrm{RC}: 1,07$, intervalle de confiance [IC] à $95 \%: 1,01$ à 1,11 ) et dans les quartiers présentant une proportion moyenne (RC : 1,09, IC à $95 \%: 1,05$ à 1,15) ou élevée de résidents souffrant d'insécurité alimentaire (RC : 1,05, IC à 95\%:1,01 à 1,11) présentaient un risque accru d'être TPAG. Le risque de naissance de nouveau-né TPAG était plus faible dans les quartiers présentant une proportion moyenne de résidents mariés (RC : 0,94, IC à $95 \%: 0,90$ à 0,98 ).

Mots-clés : faible poids à la naissance, santé fœtale, santé génésique, épidémiologie sociale, comportement sain, mode de vie sédentaire, insécurité alimentaire

\section{Introduction}

Les individus ayant eu un développement fœtal sous-optimal et dont le poids à la naissance était en conséquence faible pour l'âge gestationnel (PAG) ou très faible pour l'âge gestationnel (TPAG) présentent un risque accru de développer une maladie néonatale et sont plus susceptibles d'être atteints d'un diabète de type 2, d'hypertension, d'un syndrome métabolique et de coronopathies à l'âge adulte ${ }^{1}$.

Les facteurs de risque du développement fœtal sous-optimal englobent l'âge de la mère, l'ethnicité, la parité, l'état matrimonial, le niveau d'éducation et le tabagisme $^{1-3}$. La défavorisation du quartier est également associée à la santé ${ }^{4}$ incluant un certain nombre de facteurs de risque individuels modifiables, comme le tabagisme et la consommation d'alcool durant la grossesse ${ }^{5}$. Malheureusement, les analyses contextuelles précédentes ont souvent été fondées sur les données disponibles plutôt que sur des cheminements sociaux plausibles ${ }^{4,6}$. Au Canada et aux États-Unis, ceci a donné lieu à un ensemble de caractéristiques largement étudiées à l'échelle des quartiers et éta- blies à partir des données de recensement, dont la défavorisation économique ${ }^{7-22}$, l'origine ethnique ${ }^{10,11,15,17,19}$, la criminalité $^{15,23}$ et la présence de foyers monoparentaux $^{19}$.

Quelques études ont eu recours à des données sur des caractéristiques de l'environnement bâti et social tirées d'enquêtes spécifiques de grande envergure $^{8,11,16,24,25}$. Les chercheurs ont constaté que le faible soutien social ${ }^{24}$ et la moins bonne disponibilité en services offerts par le quartier, ou le moins grand recours à ces services ${ }^{11,16}$, étaient associés au risque d'issues défavorables de la grossesse, alors que l'environnement bâti ${ }^{16}$ et la disponibilité en restaurants et en supermarchés ${ }^{8}$ ne l'étaient pas. Le mode de vie sédentaire a aussi été associé à un risque accru de donner naissance à un nouveau-né PAG grâce à un modèle fondé sur des variables liées au contexte aux fins de santé publique ${ }^{25}$. À notre connaissance, les habitudes de consommation alimentaire des résidents n'ont pas été prises en compte dans les analyses contextuelles antérieures des nouveau-nés PAG ou TPAG.

Nous avons eu accès aux données sur les naissances simples grâce aux formulaires d'enregistrement des naissances du Québec. Nous avons recueilli les données sur les centres locaux de services communautaires (CLSC) du Québec à partir de trois sources : les formulaires d'enregistrement des naissances du Québec, une enquête sur les résidents canadiens et le recensement canadien. Nous avons formulé des hypothèses

Rattachement des auteurs :

1. Département de médecine sociale et préventive, Université Laval, Québec (Québec), Canada

2. Institut national de santé publique du Québec, Québec (Québec), Canada

3. Centre hospitalier universitaire du Québec, Québec (Québec), Canada

4. Département de mathématiques et de statistique, Université Laval, Québec (Québec), Canada

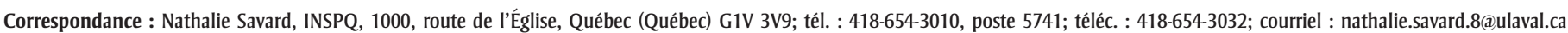


destinées à déterminer les leviers d'intervention (modèle présenté en figure 1) et analysé les liens entre la naissance de nouveau-nés TPAG et des variables individuelles liées à la mère. Nous avons aussi évalué les associations entre la naissance de nouveau-nés TPAG et des variables agrégées liées au contexte du territoire de CLSC.

\section{Méthodologie}

\section{Population à l'étude}

La population observée dans le cadre de cette étude était constituée des nouveaunés issus de naissances vivantes uniques, nés au Québec (Canada) entre 2000 et 2008, ainsi que de leur mère. Puisque les données d'enquête tirées des régions du Nord (soit le Nord-du-Québec, les TerresCries-de-la-Baie-James et le Nunavik) ne pouvaient pas être comparées à celles des autres régions de la province pour des raisons méthodologiques, nous ne les avons pas incluses. Nous avons aussi exclu les nouveau-nés dont les données sur le poids ou l'âge gestationnel étaient manquantes, ceux issus d'une grossesse de moins de 22 semaines ou de plus de 43 semaines et ceux dont le poids était invraisemblable compte tenu de l'âge gestationnel $^{26}$.

\section{Définition des territoires}

Nous avons employé les territoires des 143 CLSC du Québec. Les CLSC correspondent au premier niveau organisationnel du système de soins de santé. Ils desservent chacun en moyenne 46727 résidents et ont vu naître 4666 nouveaunés vivants uniques entre 2000 et 2008.

\section{Variables}

\section{Variable explicative}

Nous avons considéré comme nouveau-nés TPAG les nouveau-nés dont le poids à la naissance par rapport à l'âge gestationnel se situait sous le $5^{\mathrm{e}}$ percentile des courbes de croissance normalisées en fonction du sexe et de l'âge gestationnel au Canada ${ }^{27}$.

\section{Variables individuelles}

Nous avons catégorisé les caractéristiques individuelles tirées des formulaires d'enregistrement des naissances. Ces données englobaient l'âge de la mère à l'accouchement (moins de 20 ans, de 20 à 24 ans, de 25 à 29 ans, de 30 à 34 ans, 35 ans et plus); son état matrimonial (mariée dans le cadre d'une cérémonie religieuse ou civile, non mariée); le plus haut niveau d'études atteint (moins que le niveau d'études

FIGURE 1

Variables explicatives liées aux mères et aux nouveau-nés et tirées du Registre des naissances, Québec (Canada), $2000-2008$

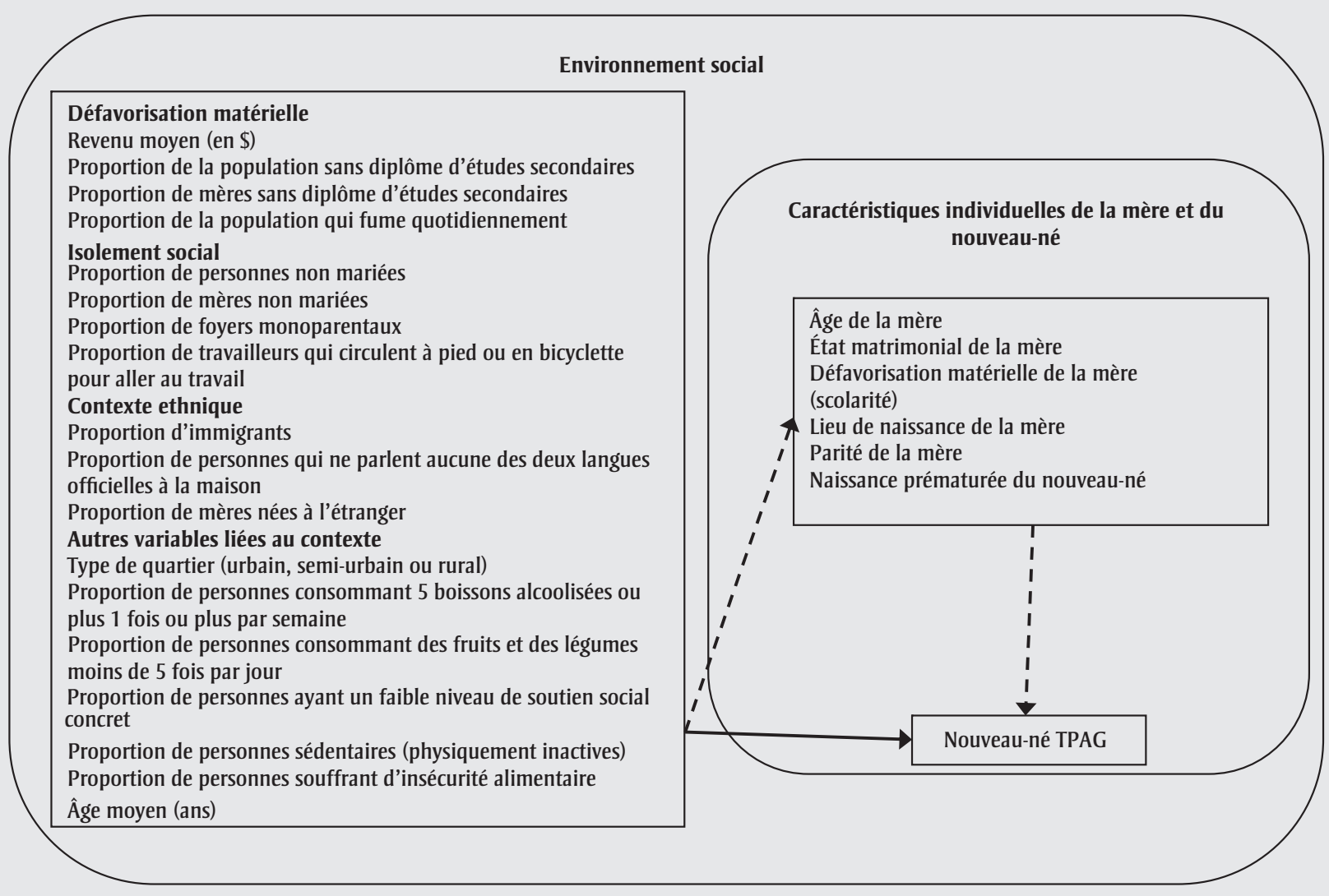

Abréviation : TPAG, très petit pour l'âge gestationnel.

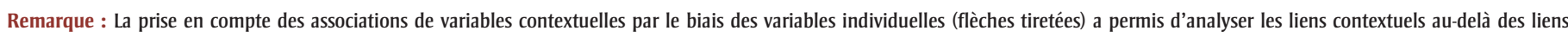
établis au moyen des variables individuelles (flèche pleine). 
secondaires, au moins un diplôme d'études secondaires, au moins un diplôme d'études collégiales, au moins un diplôme d'études universitaires); son lieu de naissance (Canada ou autre) et sa parité (primipare ou multipare).

\section{Variables agrégées liées au contexte}

Les variables agrégées liées au contexte concernant les naissances et l'ensemble de la population vivant sur le territoire d'un CLSC (hommes, autres femmes, jeunes et personnes âgées) ont permis de déterminer le niveau moyen des caractéristiques au sein de la population du territoire de ce CLSC (tableau 1). Nous avons calculé les variables agrégées concernant les naissances en agrégeant les données individuelles des CLSC. Les variables agrégées qui concernent la population ont été obtenues à la fois en calculant des proportions à partir des réponses données par les répondants de l'Enquête sur la santé dans les collectivités canadiennes (ESCC) ${ }^{25,28}$ et en regroupant des profils de recensement à l'échelle des sous-territoires. Les proportions ont été regroupées en premier, deuxième et troisième tertiles (parties inférieure, moyenne et supérieure de la distribution). Le premier tertile a servi de référence pour toutes les variables, à l'exception du revenu moyen, pour lequel la référence était le troisième tertile.

Nous avons imputé les valeurs manquantes au moyen de la procédure d'imputation multiple ("MI ») du logiciel SAS, en utilisant la méthode " MCMC » pour les variables individuelles par catégorie et l'algorithme «EM » avec transformation logit pour les proportions ${ }^{29}$.

\section{Sources des données}

Les formulaires d'enregistrement des naissances de 2000 à 2008 ont été tirés du registre des événements démographiques du Québec ${ }^{30}$. Ils contiennent des données sur toutes les naissances vivantes (poids à la naissance, âge de la mère à l'accouchement, état matrimonial, plus haut niveau d'études atteint par la mère, lieu de naissance de la mère, parité) et le code postal de résidence de la mère au moment de l'accouchement.

L'ESCC est une enquête transversale ayant été menée, jusqu'à présent, sur quatre cycles d'un an (2000-2001, 2003, 2005 et 2007-2008) ${ }^{28}$. Pour accroître la puissance statistique, nous avons regroupé les données de ces quatre enquêtes annuelles ${ }^{31}$.

Les profils de recensement de 2001 et de 2006 sont disponibles à une échelle plus fine que celle des territoires des CLSC, soit les secteurs de recensement (pour les régions métropolitaines) et les subdivisions (partout ailleurs). Ils constituent les

TABLEAU 1

Variables contextuelles explicatives au niveau des centres locaux de services communautaires (CLSC), Québec (Canada), 2000-2008

\begin{tabular}{|c|c|}
\hline Population cible et source de données & Variable contextuelle \\
\hline \multicolumn{2}{|l|}{ Information du Registre des naissances (2000-2008) } \\
\hline Naissances & Mères sans diplôme d'études secondaires (\%) \\
\hline Naissances & Mères nées à l'étranger (\%) \\
\hline Naissances & Mères non mariées (\%) \\
\hline \multicolumn{2}{|c|}{ Enquête sur la santé dans les collectivités canadiennes (2000-2001; 2003; 2005; 2007-2008) } \\
\hline Population de 12 ans et plus ${ }^{\mathrm{a}}$ & Personnes fumant quotidiennement $(\%)^{b}$ \\
\hline Population de 12 ans et plus ${ }^{\mathrm{a}}$ & Personnes consommant 5 boissons alcoolisées ou plus par occasion, 1 fois ou plus par semaine $(\%)^{b}$ \\
\hline Population de 12 ans et plus ${ }^{a}$ & Personnes consommant des fruits et des légumes moins de cinq fois par jour (\%) \\
\hline Population de 12 ans et plus ${ }^{\mathrm{a}}$ & Personnes ayant un faible niveau de soutien social concret $(\%)^{b, c}$ \\
\hline Population de 12 ans et plus ${ }^{\mathrm{a}}$ & Personnes sédentaires (physiquement inactives) au cours des 3 derniers mois (\%) \\
\hline Population de 12 ans et plus ${ }^{\mathrm{a}}$ & Personnes ayant souffert d'insécurité alimentaire au cours des 12 derniers mois (\%) \\
\hline \multicolumn{2}{|l|}{ Profils de recensement (2000 et 2006) } \\
\hline Population totale & $\begin{array}{l}\text { Continuum urbain-rural (centre local de services communautaires combinant seulement des sous- } \\
\text { territoires urbains, seulement des sous-territoires ruraux ou les deux) }\end{array}$ \\
\hline $\begin{array}{l}\text { Population de } 25 \text { à } 64 \text { ans (2006) ou de } 20 \text { ans et plus } \\
\text { (2001) }\end{array}$ & Personnes sans diplôme d'études secondaires (\%) \\
\hline Population totale & Âge moyen (ans) \\
\hline Population totale & Immigrants (\%) \\
\hline Population totale & Personnes qui ne parlent aucune des deux langues officielles à la maison (\%) \\
\hline Population de 15 ans et plus avec revenu & Revenu moyen (\$) \\
\hline Population de 15 ans et plus & Personnes non mariées (\%) \\
\hline Foyers (privés) & Foyers monoparentaux (\%) \\
\hline Travailleurs de 15 ans et plus & Travailleurs qui circulent à pied ou en vélo pour aller au travail (\%) \\
\hline
\end{tabular}

a Englobe seulement les personnes vivant au domicile familial.

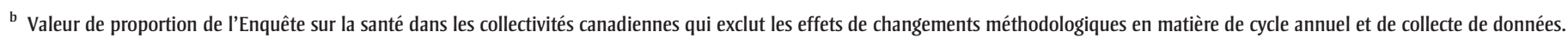

c Moins de 15 sur 95 sur la sous-échelle Social Support Survey de la Medical Outcomes Study. 
sous-territoires de notre étude. La taille des populations de chacun de ces sousterritoires est similaire. Les profils infraterritoriaux ont été agrégés par CLSC, sans tenir compte de l'année de collecte des données.

\section{Analyse statistique}

Les valeurs liées au contexte sur les territoires de CLSC ont été associées aux naissances individuelles en fonction du code postal de résidence des mères. Des rapports de cotes (RC) ont été utilisés pour évaluer les risques relatifs.

\section{Régression}

Nous avons évalué les RC ajustés pour les variables individuelles ( $\mathrm{RC}_{\text {ajustés }}^{\mathrm{I}}$ au moyen de modèles de régression logistique multiniveau ajustés à l'aide d'équations d'estimation généralisées (EEG). Nous avons choisi la mère comme premier niveau et le CLSC comme second niveau $^{33}$. Les EEG ont fourni des estimations de RC appropriées pour faire de l'inférence au niveau populationnel, et ce, même si la corrélation entre les mères relevant du même CLSC était inconnue. Nous avons considéré que cette corrélation était faible, et la matrice de " corrélation de travail indépendante » a servi de point de départ pour amorcer les calculs. Nous avons obtenu des estimations empiriques des erreurs-types, ce qui nous a permis d'éviter des erreurs qui auraient pu être causées par un mauvais choix de matrice de corrélation de travail $^{34}$.

Un test de déviance a été réalisé pour déterminer si les CLSC permettaient d'expliquer une partie importante de la variabilité inexpliquée par le modèle individuel à termes d'interaction.

Nous avons obtenu des RC bruts ( $\mathrm{RC}^{\mathrm{E}}$ bruts) et des RC ajustés pour les variables individuelles et des termes d'interaction $\left(\mathrm{RC}^{\mathrm{E}}\right.$ ajustés I $)$ au moyen de la méthode EEG pour chacune des variables liées au contexte. Les interactions entre les variables individuelles ont été sélectionnées à l'aide d'une méthode pas-à-pas utilisant l'option " hierarchy = multiple » de la procédure logistique (valeurs $p$ d'entrée/séjour inférieures à 0,001). Le modèle final a été conçu au moyen d'une méthode pas-à-pas qui forçait l'intégration des variables individuelles et des termes d'interaction. Ce modèle avait comme variables candidates les variables liées au contexte ayant des valeurs de $\mathrm{RC}^{\mathrm{E}}$ ajustés I significatives (valeurs $p$ d'entrée/séjour de 0,25 et 0,05 respectivement). Des estimations de paramètres EEG ajustées pour les variables individuelles et pour d'autres variables liées au contexte $\left(\mathrm{RC}^{\mathrm{E}}\right.$ ajustés IE $)$ ont été produites pour chacune des variables liées au contexte.

Les résultats liés au contexte étaient limités à la présentation de ces variables et des différences au niveau des RC bruts. Nous avons présenté seulement une variable de défavorisation matérielle, de contexte ethnique et d'isolement social (figure 1) par ensemble de données (toutes les données peuvent être fournies par les auteurs sur demande).

Les valeurs de $\mathrm{RC}$ ajustés ( $\mathrm{RC}_{\text {ajustés, }}^{\mathrm{I}}$ $R C^{\mathrm{E}}$ ajustés I et $R C^{\mathrm{E}}$ ajustés IE) ont été validées par le biais de deux analyses de sensibilité. La première a tenu compte des données non imputées, alors que la deuxième a intégré des variables à l'échelle territoriale la plus fine, c'est-à-dire les données de recensement et les données sur les naissances au niveau des 2368 sous-territoires, de même que les données tirées de l'ESCC au niveau des CLSC.

La Commission d'accès à l'information du Québec et le Comité d'éthique de l’Université Laval ont approuvé ce projet de recherche. Nous avons procédé aux analyses à l'aide du logiciel SAS, version 9.2 (procédures " MI », " LOGISTIC » et «GENMOD ») ${ }^{29}$. Les résultats de l'analyse de régression ont été jugés statistiquement significatifs lorsque les valeurs $p$ étaient inférieures à 0,05.

\section{Résultats}

\section{Analyse descriptive}

Parmi les 676165 bébés issus de naissances uniques enregistrés dans toutes les régions du Québec entre 2000 et 2008, 7379 étaient nés de mères vivant dans une région du Nord, 850 n’ont pas pu être liés à un CLSC, 67 n'ont pas pu obtenir un statut de poids pour l'âge gestationnel (données manquantes sur le poids ou l'âge gestationnel), 452 étaient issus d'une grossesse de moins de 22 semaines ou de plus de 43 semaines et 163 avaient un poids invraisemblable compte tenu de leur âge gestationnel. Notre population comptait donc 667254 nouveau-nés vivant sur 143 CLSC.

\section{Régression}

Un lien significatif a été établi entre chaque variable individuelle et la naissance d'un nouveau-né TPAG (tableau 2). Les mères ne possédant pas de diplôme d'études secondaires, celles qui en avaient obtenu un et celles qui avaient obtenu un diplôme d'études collégiales avaient un risque accru de donner naissance à un nouveau-né TPAG ( $\mathrm{RC}_{\text {ajustés }}^{\mathrm{I}}$ : 2,08, 1,53 et 1,14 , respectivement) comparativement aux mères ayant obtenu au moins un diplôme d'études universitaires. Les mères primipares présentaient également un risque plus élevé $\left(\mathrm{RC}^{\mathrm{I}}{ }_{\text {ajusté }}: 1,96\right)$ que les autres femmes, toutes les autres variables individuelles étant égales par ailleurs.

Les territoires de CLSC représentaient une partie importante de la variation inexpliquée découlant du modèle individuel incorporant des termes d'interaction (valeur du chi carré : 497,3, $p<0,001$; degrés de liberté : 142). Il était donc approprié d'inclure les variables agrégées par CLSC dans le modèle.

On a observé des associations brutes entre les nouveau-nés TPAG et toutes les variables liées au contexte présentées, à l'exception des variables " personnes consommant des fruits et des légumes moins de 5 fois par jour » et " continuum urbain-rural » (tableau 3; d'autres données peuvent être fournies par les auteurs sur demande). Les RC ajustés ( $\mathrm{RC}^{\mathrm{E}}$ ajustés I) étaient légèrement inférieurs aux valeurs brutes ( $\mathrm{RC}^{\mathrm{E}}$ bruts), bien que les intervalles de confiance n'aient indiqué aucune différence significative. En tenant compte des variables individuelles, les naissances des CLSC du revenu moyen le plus faible $\left(\mathrm{RC}^{\mathrm{E}}\right.$ ajusté $\left.\mathrm{I}: 1,12\right)$ et les variables se situant au troisième tertile des catégories suivantes présentaient un risque accru de naissances de bébés TPAG : des mères 
TABLEAU 2

Rapports de cotes ajustés pour les naissances vivantes uniques de nouveau-nés TPAG, d'après certaines variables explicatives individuelles liées aux mères, Québec (Canada), 2000-2008

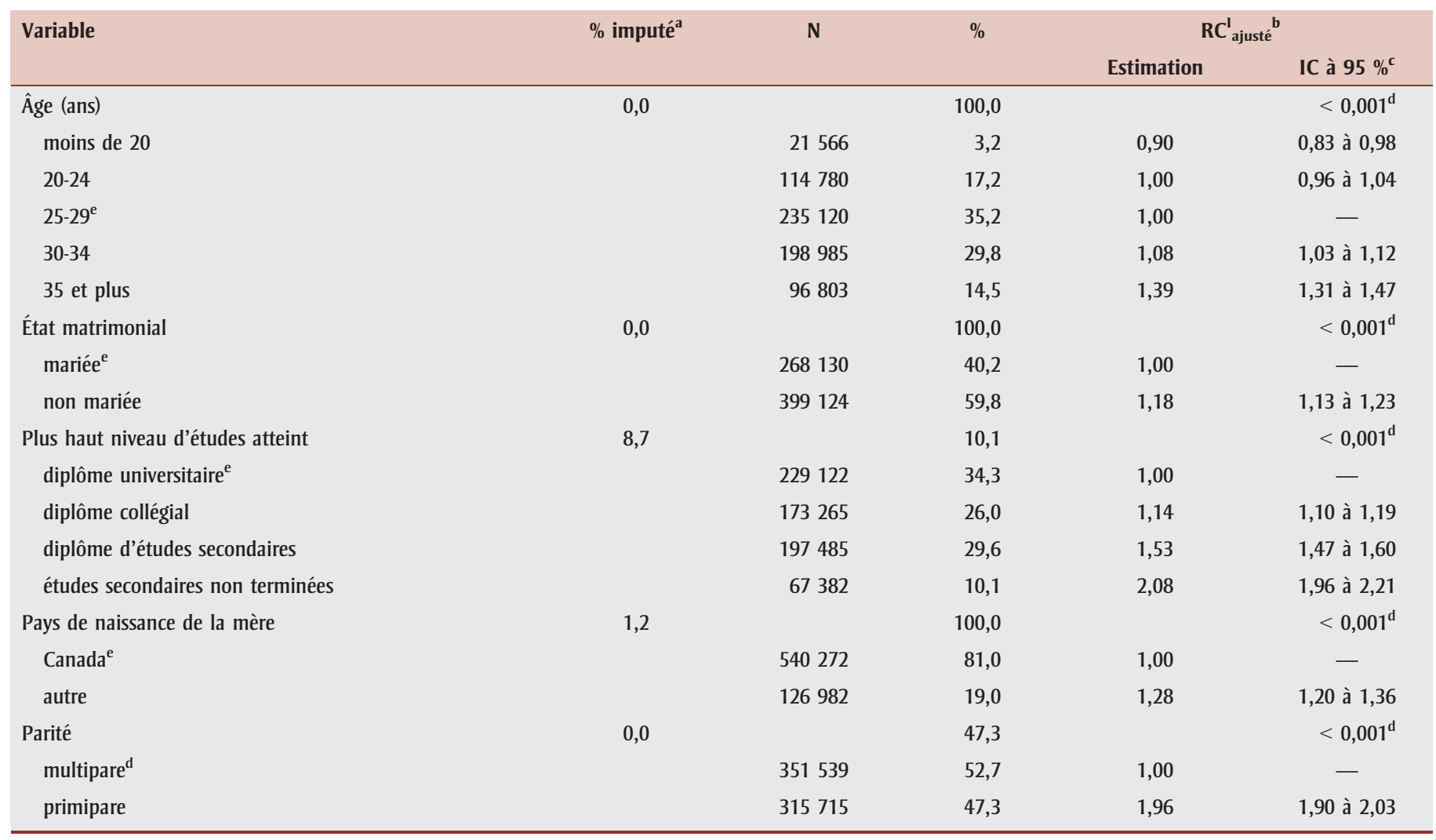

Abréviations: IC, intervalle de confiance; RC, rapport de cotes; TPAG, très petit pour l'âge gestationnel.

a Pourcentage de naissances auxquelles les valeurs ont été imputées.

b Rapport de cotes ajusté pour les variables individuelles (âge de la mère, état matrimonial de la mère, niveau d'études de la mère, pays de naissance de la mère et parité de la mère).

c Intervalles de confiance calculés au moyen d'estimations de variance robustes tirées d'un modèle multiniveau ajusté selon des équations d'estimation généralisées (EEG).

${ }^{\mathrm{d}}$ Valeur $p$ servant à évaluer les différences au sein de l'ensemble.

e Catégorie de référence.

sans diplôme d'études secondaires $\left(\mathrm{RC}^{\mathrm{E}}\right.$ ajusté $\left.\mathrm{I}: 1,12\right)$; des mères immigrantes $\left(\mathrm{RC}^{\mathrm{E}}\right.$ ajusté I : 1,06); des mères nées à l'étranger $\left(\mathrm{RC}^{\mathrm{E}}\right.$ ajusté I : 1,08); des personnes ne parlant aucune des deux langues officielles à la maison $\left(\mathrm{RC}^{\mathrm{E}}\right.$ ajusté $\left.\mathrm{I}: 1,08\right)$ et des personnes dans un foyer monoparental $\left(\mathrm{RC}^{\mathrm{E}}\right.$ ajusté I : 1,11) (tableau 3). Les naissances au sein de CLSC se situant aux deuxième et troisième tertiles de l'insécurité alimentaire $\left(\mathrm{RC}^{\mathrm{E}}\right.$ ajustés I $: 1,08$ et 1,14$)$ et de la sédentarité $\left(\mathrm{RC}^{\mathrm{E}}\right.$ ajustés I $: 1,06$ et 1,11$)$ présentaient aussi des risques accrus de TPAG, alors que les naissances au sein de CLSC se situant au deuxième tertile pour la variable « résidents non mariés » $\left(\mathrm{RC}^{\mathrm{E}}\right.$ ajusté $\mathrm{I}$ : 0,93 ) présentaient des risques plus faibles.

Le modèle final intégrait des variables liées au contexte: l'insécurité alimentaire, la sédentarité et l'état matrimonial. Les naissances au sein des CLSC se situant au deuxième ou troisième tertile d'insécurité alimentaire étaient exposées à un risque plus élevé de TPAG $\left(\mathrm{RC}^{\mathrm{E}}\right.$ ajustés IE : 1,05; $1,09)$ après ajustement pour toutes les variables individuelles, pour les résidents non mariés et pour la sédentarité. Les naissances au sein des CLSC se situant au troisième tertile de sédentarité étaient aussi exposées à un risque plus élevé de TPAG $\left(\mathrm{RC}^{\mathrm{E}}\right.$ ajusté IE $\left.: 1,07\right)$ après ajustement pour ces mêmes variables. De façon similaire, les naissances dans des CLSC ayant une proportion moyenne de résidents non mariés couraient un risque plus faible de TPAG (RCE ${ }_{\text {ajusté IE }}$ : 0,94) (tableau 3).

Les RC ajustés $\left(R C^{\mathrm{I}}\right.$ ajustés, $R C^{\mathrm{E}}$ ajustés I et $\mathrm{RC}^{\mathrm{E}}$ ajustés IE) auraient été similaires si nous avions utilisé des données non imputées. Certaines valeurs de $\mathrm{RC}^{\mathrm{I}}$ ajustés (pour les mères de 35 ans et plus, pour les mères ayant un diplôme d'études secondaires ou moins, de même que pour les mères primipares) auraient été plus petites, et les $\mathrm{RC}^{\mathrm{E}}$ ajustés I et les $\mathrm{RC}^{\mathrm{E}}$ ajustés IE auraient été similaires si nous avions examiné les $5^{\mathrm{e}}$ à $10^{\mathrm{e}}$ percentiles des poids à la naissance. De même, les $\mathrm{RC}^{\mathrm{E}}$ ajustés I et les $\mathrm{RC}^{\mathrm{E}}$ ajustés IE auraient été semblables s'ils avaient été évalués au moyen d'un modèle logistique intégrant des variables au niveau territorial le plus fin. Les seules exceptions auraient concerné le troisième tertile des mères sans diplôme d'études secondaires et le deuxième tertile des foyers monoparentaux, pour lesquels les valeurs de $\mathrm{RC}^{\mathrm{E}}$ ajustés I auraient été supérieures dans la dernière analyse. 
TABLEAU 3

Rapports de cotes bruts et ajustés pour les naissances vivantes uniques de nouveau-nés TPAG, d'après certaines variables contextuelles, Québec (Canada), 2000-2008

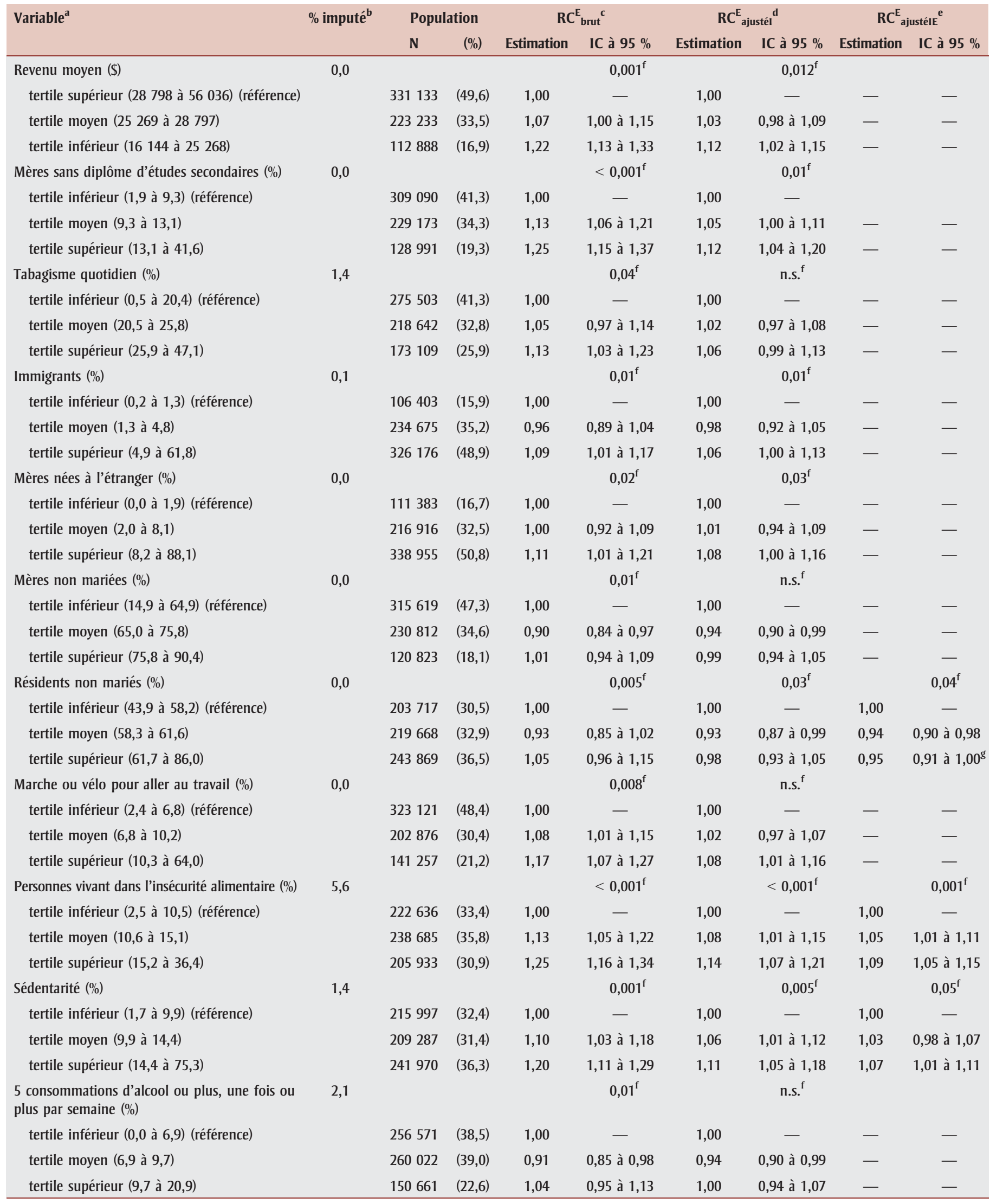


TABLEAU 3 (Suite)

Rapports de cotes bruts et ajustés pour les naissances vivantes uniques de nouveau-nés TPAG, d'après certaines variables contextuelles, Québec (Canada), 2000-2008

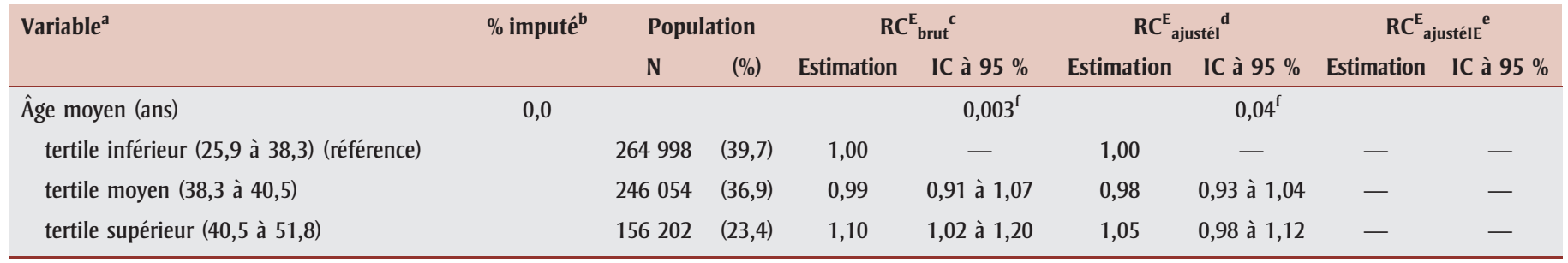

Abréviations : IC, intervalle de confiance; n.s., non significatif; RC, rapport de cotes; TPAG, très petit pour l'âge gestationnel.

Remarque : intervalle de confiance calculé au moyen d'estimations de variance robustes tirées d'un modèle multiniveau ajusté selon des équations d'estimation généralisée (EEG).

a L'interprétation d'un résultat contextuel comme substitut de la variable individuelle correspondante pourrait être inappropriée.

b Pourcentage des centres locaux de services communautaires dont les valeurs ont été imputées.

c Rapport de cotes brut.

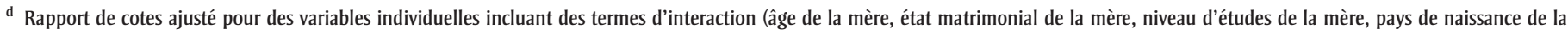
mère, parité de la mère, niveau d'études $\times$ état matrimonial, pays de naissance $\times$ âge, pays de naissance $\times$ état matrimonial, niveau d'études $\times$ pays de naissance, âge $\times$ parité et niveau d'études $\times$ parité).

e Rapport de cotes ajusté pour des variables contextuelles (personnes souffrant d'insécurité alimentaire et personnes inactives) et pour des variables individuelles incluant des termes d'interaction.

${ }^{\mathrm{f}}$ Valeur $p$ servant à évaluer les différences dans l'ensemble.

${ }^{8}$ Valeur $<1,0$.

\section{Analyse}

Nous avons adopté une approche globale pour comprendre les déterminants de la santé fœtale au Québec (Canada) en utilisant l'information liée au contexte tirée d'une enquête spécifique, les données sur les naissances et les données de recensement dans un contexte où les données individuelles étaient disponibles. Nous avons trouvé des liens entre les naissances de nouveau-nés TPAG et des variables liées au contexte de chaque source de données indépendamment des variables individuelles. Ni les données de recensement, ni les données d'enquête, ni celles du Registre des naissances du Québec ne contenaient à elles seules un éventail aussi large de variables liées au contexte. Les variables d'insécurité alimentaire et de sédentarité étaient pertinentes pour l'inclusion dans un modèle à plusieurs variables liées au contexte. Elles se sont révélées toutes deux associées de façon significative à la survenue de TPAG. Notons que ces variables agrégées ne reflètent pas nécessairement l'insécurité alimentaire et la sédentarité individuelle. Par exemple, dans une analyse précédente ${ }^{25}$, la proportion de résidents vivant sous le seuil de faible revenu sur le territoire de CLSC était corrélée à l'isolement social et au contexte ethnique, alors que le revenu moyen sur ce même territoire était lié à la défavorisation matérielle.

Certaines variables liées au contexte analysées dans le cadre de cette étude ont également été intégrées dans d'autres études canadiennes et américaines ${ }^{7,9,13,14,19}$. Lorsque des variables individuelles et certaines variables liées au contexte étaient prises en compte, des liens significatifs ont été établis entre des naissances de nouveaunés PAG et le seuil de faible revenu pour les naissances survenues au Québec de 1991 à $2000^{14}$ et celles survenues à Montréal de 1997 à $2001^{9}$. De plus, un lien significatif a été observé entre les naissances de bébés PAG et la défavorisation matérielle mesurée par le revenu régional en Ontario entre 2004 et $2006^{13}$.

L'isolement social et le contexte ethnique (mesurés par le nombre de foyers monoparentaux, le faible revenu et l'ethnicité) n’ont pas été associés de façon significative à un poids faible à la naissance chez les nouveau-nés de Caroline du Sud entre 2000 et $2003^{19}$, lorsqu'on a tenu compte des variables individuelles et de plusieurs variables liées au contexte. Ces variables n'ont pas été ajoutées à notre modèle final à plusieurs variables liées au contexte.

\section{Limites}

Il convient de souligner quelques limites à cette étude. D’abord, nous nous sommes penchés sur le développement fœtal sousoptimal mesuré par l'indicateur TPAG. La petite constitution de certains nouveaunés pourrait ne pas être attribuable à un développement fœtal sous-optimal, mais ceux-ci pourraient quand même avoir été désignés comme nouveau-nés TPAG, ce qui expliquerait un biais de classification non différentielle dans le résultat. Une telle classification erronée a été minimisée par l'emploi de l'indicateur TPAG plutôt que celui de l'indicateur PAG.

L'attribution de catégories d'exposition aux CLSC a pu être faite de façon erronée. En regroupant les données, nous avons implicitement fait le postulat que les tertiles des CLSC sont demeurés les mêmes tout au long des années. De plus, l'information concernant les mères ayant déménagé n'était pas disponible. D'après les données du Recensement de $2006^{35}$, environ $3,5 \%$ des femmes n'ont pas été correctement assignées au tertile de CLSC que nous leur avions attribué, ces erreurs de classification ayant contribué à créer un léger biais en faveur d'un résultat nul. 
Des risques de confusion dans nos résultats ont pu relever de facteurs individuels non mesurés, comme certaines caractéristiques maternelles liées à l'isolement social, au mode de vie (tabagisme, consommation de caféine, consommation élevée d'alcool, sédentarité ou survenue d'abus physique) et à l'état de santé (apport calorique quotidien, indice de masse corporelle de la mère, hypertension de la mère ou diabète de grossesse).

Nos données agrégées ne nous ont pas permis de distinguer l'effet d'une exposition contextuelle durant la grossesse à une exposition antérieure, ni de déterminer si le lien entre le contexte et la survenue de naissances TPAG a changé au fil du temps.

Enfin, notre étude a été limitée en raison du manque de connaissances à propos de l'échelle spatiale appropriée pour étudier la santé fœtale $e^{36}$. Des analyses de sensibilité ont d'ailleurs été fondées sur les données regroupées par sous-territoires.

Les mécanismes via lesquels l'insécurité alimentaire pourrait être associée au poids plus faible à la naissance selon l'âge gestationnel englobent les facteurs interpersonnels, pour lesquels un lien systématique avec certains comportements alimentaires a été établi chez les jeunes ${ }^{37}$. Un poids plus élevé chez les mères avant la grossesse, facteur qui n'a pas pu être mesuré, pourrait aussi entraîner un diabète gestationnel ${ }^{38}$.

Les résidents des CLSC avec une moindre sédentarité et une moindre inactivité sont certainement en meilleure santé, et leur risque de développer des maladies chroniques et de souffrir d'incapacité est plus faible $^{39}$. Les mères vivant sur ces territoires de CLSC ont plus de chances d'être physiquement actives. L'activité chez les résidents de ces CLSC pourrait être attribuable à un environnement bâti favorisant d'une manière ou d'une autre la pratique d'activités ${ }^{37,39,40}$ plutôt qu'à un environnement social ${ }^{37}$ faisant de même. De plus, être actif est le signe d'une compréhension générale des messages de santé publique (manger mieux, faire de l'exercice, ne pas fumer, etc. $)^{37}$. Nos résultats semblent pertinents pour d'autres pays dotés de systèmes de bien-être social comparables.
Dans notre but d'englober d'avantage de déterminants contextuels de la santé fœtale, nous avons intégré des données agrégées tirées d'une enquête canadienne de même que des données de recensement et des données sur les naissances, de manière à établir des profils diversifiés des communautés. Ces profils, établis à partir d'un large éventail de variables, nous ont permis de mettre en évidence des liens contextuels entre les naissances d'enfants TPAG et l'état matrimonial, l'insécurité alimentaire et la sédentarité des résidents. Ces liens contextuels n’ont pas été désignés comme " associations contextuelles » en tant que tel, car aucun ajustement n'a été réalisé pour l'insécurité alimentaire et la sédentarité individuelle des mères dans les analyses, même si plusieurs ajustements ont été faits pour d'autres caractéristiques individuelles.

Les résultats de cette étude s'ajoutent au nombre croissant de données probantes qui laissent penser que des processus sociaux contextuels affectent la santé fœtale. Des études canadiennes ultérieures pourraient tirer profit de l'information recueillie dans le cadre d'enquêtes de grande envergure, notamment dans le cadre de l'ESCC, en l'intégrant à l'ensemble restreint des données de recensement, de manière à brosser un tableau des différents quartiers et à utiliser les données détaillées ainsi obtenues dans le cadre d'une approche globale.

\section{Références}

1. Organisation mondiale de la Santé. Pour un développement optimal du fœus : rapport d'une consultation technique, Genève $(\mathrm{CH})$ : Éditions de l’OMS; 2006.

2. Henrichs J, Schenk JJ, Roza SJ et collab. Maternal psychological distress and fetal growth trajectories: the Generation R Study. Psychol Med. 2010;40:633-43.

3. Blumenshine P, Egerter S, Barclay CJ, Cubbin C, Braveman PA. Socioeconomic disparities in adverse birth outcomes: a systematic review. Am J Prev Med. 2010;39:263-72.

4. Berkman LF, Kawachi I. Social Epidemiology. New York (NY) : Oxford University Press; 2000.
5. Timmermans S, Bonsel GJ, SteegersTheunissen RP et collab. Individual accumulation of heterogeneous risks explains perinatal inequalities within deprived neighbourhoods. Eur J Epidemiol. 2010; 26:165-80.

6. Riva M, Gauvin L, Barnett TA. Toward the next generation of research into small area effects on health: a synthesis of multilevel investigations published since July 1998. J Epidemiol Community Health. 2007;61: 853-61.

7. Auger N, Giraud J, Daniel M. The joint influence of area income, income inequality, and immigrant density on adverse birth outcomes: a population-based study. BMC Public Health. 2009;9:237.

8. Farley TA, Mason K, Rice J, Habel JD, Scribner R, Cohen DA. The relationship between the neighbourhood environment and adverse birth outcomes. Paediatr Perinat Epidemiol. 2006;20:188-200.

9. Généreux M, Auger N, Goneau M, Daniel M. Neighbourhood socioeconomic status, maternal education and adverse birth outcomes among mothers living near highways. J Epidemiol Community Health. 2008;62:695-700.

10. Gorman BK. Racial and ethnic variation in low birthweight in the United States: individual and contextual determinants. Health Place. 1999;5:195-207.

11. Jaffee KD, Perloff JD. An ecological analysis of racial differences in low birthweight: implications for maternal and child health social work. Health Soc Work. 2003;28: $9-22$.

12. Krieger N, Chen JT, Waterman PD, Rehkopf DH, Subramanian SV. Painting a truer picture of US socioeconomic and racial/ ethnic health inequalities: the Public Health Disparities Geocoding Project. Am J Public Health. 2005;95:312-23.

13. Liu N, Wen SW, Katherine W, Bottomley J, Yang Q, Walker MC. Neighbourhood family income and adverse birth outcomes among singleton deliveries. J Obstet Gynaecol Can. 2010;32:1042-8.

14. Luo ZC, Wilkins R, Kramer MS. Effect of neighbourhood income and maternal education on birth outcomes: a populationbased study. JAMC. 2006;174:1415-20. 
15. Masi CM, Hawkley LC, Piotrowski ZH, Pickett KE. Neighborhood economic disadvantage, violent crime, group density, and pregnancy outcomes in a diverse, urban population. Soc Sci Med. 2007;65:2440-57.

16. Muhajarine N, Vu LT. Neighbourhood contexts and low birthweight: social disconnection heightens single parents risks in Saskatoon. Can J Public Health. 2009;100:130-4.

17. Nkansah-Amankra S, Luchok KJ, Hussey JR, Watkins K, Liu X. Effects of maternal stress on low birth weight and preterm birth outcomes across neighborhoods of South Carolina, 2000-2003. Matern Child Health J. 2010;14:215-26.

18. Nkansah-Amankra S, Dhawain A, Hussey JR, Luchok KJ. Maternal social support and neighborhood income inequality as predictors of low birth weight and preterm birth outcome disparities: analysis of South Carolina pregnancy risk assessment and monitoring system survey, 2000-2003. Matern Child Health J. 2010;14:774-85.

19. Nkansah-Amankra S. Neighborhood contextual factors, maternal smoking, and birth outcomes: multilevel analysis of the South Carolina PRAMS survey, 2000-2003. J Womens Health (Larchmt). 2010;19: 1543-52.

20. Pearl M, Braveman P, Abrams B. The relationship of neighborhood socioeconomic characteristics to birthweight among 5 ethnic groups in California. Am J Public Health. 2001;91:1808-14.

21. Rich-Edwards JW, Buka SL, Brennan RT, Earls F. Diverging associations of maternal age with low birthweight for black and white mothers. Int J Epidemiol. 2003;32: 83-90.

22. Subramanian SV, Chen JT, Rehkopf DH, Waterman PD, Krieger N. Comparing individual- and area-based socioeconomic measures for the surveillance of health disparities: a multilevel analysis of Massachusetts births, 1989-1991. Am J Epidemiol. 2006;164:823-34.

23. Messer LC, Kaufman JS, Dole N, Herring A, Laraia BA. Violent crime exposure classification and adverse birth outcomes: a geographically-defined cohort study. Int J Health Geogr. 2006;5:22.
24. Buka SL, Brennan RT, Rich-Edwards JW, Raudenbush SW, Earls F. Neighborhood support and the birth weight of urban infants. Am J Epidemiol. 2003;157:1-8.

25. Savard N, Levallois P, Rivest LP, Gingras S. A study of the association between characteristics of the CLSCs and the risk of small for gestational age births among term and preterm births in Quebec, Canada. Can J Public Health. 2012;103:152-7.

26. Alexander GR, Himes JH, Kaufman RB et collab. A United States national reference for fetal growth. Obstet Gynecol. 1996;87: 163-8.

27. Kramer MS, Platt RW, Wen SW et collab. A new and improved population-based Canadian reference for birth weight for gestational age. Pediatrics. 2001;108(2): E35.

28. Béland Y. Enquête sur la santé dans les collectivités canadiennes: aperçu de la méthodologie. Rapport sur la santé. 2002;13:9-15.

29. SAS Institute Inc. SAS/STAT 9.2 user's guide: the MI procedure. $2^{\mathrm{e}}$ éd. Cary (NC) : SAS Institute Inc; 2009.

30. Ministère de la Santé et des Services sociaux. Registre des événements démographiques - Fichier des naissances vivantes (RED/naissances vivantes (K29)) [Internet]. Québec (Qc): gouvernement du Québec; [consultation le 10 février 2011]. Consultable en ligne à partir de la page: http://www.informa.msss.gouv.qc.ca/ Details.aspx? $\mathrm{Id}=\mathrm{pDH} 1 \mathrm{q} 4 \mathrm{exKSc}=$ \&Source $=$ $/ \mathrm{dlVmYIVYBQ}=$

31. Thomas S, Wannell B. Combiner les cycles de l'Enquête sur la santé dans les collectivités canadiennes. Rapport sur la santé. 2009;20:59-65.

32. Statistique Canada. Profil pour le Canada, les provinces, les territoires, les divisions de recensement et les subdivisions de recensement, Recensement de 2006 [Internet]. Ottawa (Ont.) : Statistique Canada; [consultation le 10 février 2011]. Consultable en ligne à partir de la page: http://www5.statcan.gc.ca/bsolc/olc-cel/olccel?catno $=94-581-\mathrm{X} 2006001$ \&lang $=$ fra
33. Rothman KJ, Greenland S, Lash TL. Modern epidemiology. $3^{\mathrm{e}}$ éd. Philadelphia (PA) : Wolters Kluwer Health/Lippincott Williams \& Wilkins; 2008.

34. Hardin JW, Hilbe JM. Generalized estimating equations. Boca Raton (FL) : Chapman \& Hall/CRC; 2002.

35. Statistique Canada. Population âgée de 5 ans et plus selon la mobilité, par province et territoire (Recensement de 2006) [Internet]. Ottawa (Ont.) : Statistique Canada; [consultation le 31 juillet 2013]. Consultable à partir de la page: http:// www.statcan.gc.ca/tables-tableaux/sum-som /102/cst01/demo56b-fra.htm

36. Diez Roux AV. Neighborhoods and health: where are we and where do we go from here? Rev Epidemiol Sante Publique. 2007;55:13-21.

37. De Vet E, de Ridder DT, de Wit JB. Environmental correlates of physical activity and dietary behaviours among young people: a systematic review of reviews. Obes Rev. 2011;12:e130-42.

38. Janevic T, Borrell LN, Savitz DA, Herring AH, Rundle A. Neighbourhood food environment and gestational diabetes in New York City. Paediatr Perinat Epidemiol. 2010;24:249-54.

39. Haskell WL, Blair SN, Hill JO. Physical activity: health outcomes and importance for public health policy. Prev Med. 2009;49:280-2.

40. Durand CP, Andalib M, Dunton GF, Wolch J, Pentz MA. A systematic review of built environment factors related to physical activity and obesity risk: implications for smart growth urban planning. Obes Rev. 2011;12:e173-82. 